Draft VERSion June 21, 2021

Typeset using LATEX twocolumn style in AASTeX61

\title{
MODELING THE MULTI-BAND AFTERGLOWS OF GRB 060614 AND GRB 060908: FURTHER EVIDENCE FOR A DOUBLE POWER-LAW HARD ELECTRON ENERGY SPECTRUM
}

\author{
Q. ZhANG, ${ }^{1}$ S. L. XIONG, ${ }^{1}$ AND L. M. SONG ${ }^{1}$ \\ ${ }^{1}$ Key Laboratory of Particle Astrophysics, Institute of High Energy Physics, Chinese Academy of Sciences, Beijing 100049, China \\ (Received; Revised; Accepted) \\ Submitted to ApJ \\ ABSTRACT
}

\begin{abstract}
Electrons accelerated in relativistic collisionless shocks are usually assumed to follow a power-law energy distribution with an index of $p$. Observationally, although most gamma-ray bursts (GRBs) have afterglows that are consistent with $p>2$, there are still a few GRBs suggestive of a hard $(p<2)$ electron energy spectrum. Our previous work showed that GRB 091127 gave strong evidence for a double power-law hard electron energy (DPLH) spectrum with $1<p_{1}<2, p_{2}>2$ and an "injection break" assumed as $\gamma_{\mathrm{b}} \propto \gamma^{q}$ in the highly relativistic regime, where $\gamma$ is the bulk Lorentz factor of the jet. In this paper, we show that GRB 060614 and GRB 060908 provide further evidence for such a DPLH spectrum. We interpret the multi-band afterglow of GRB 060614 with the DPLH model in an homogeneous interstellar medium by taking into account a continuous energy injection process, while for GRB 060908, a wind-like circumburst density profile is used. The two bursts, along with GRB 091127, suggest a similar behavior in the evolution of the injection break, with $q \sim 0.5$. Whether this represents a universal law of the injection break remains uncertain and more such afterglow observations are needed to test this conjecture.
\end{abstract}

Keywords: acceleration of particles - gamma-ray burst: individual (GRB 060614, GRB 060908) radiation mechanisms: non-thermal 


\section{INTRODUCTION}

Gamma-Ray bursts (GRBs) are the most energetic stellar explosions in the universe. They produce a short prompt $\gamma$-ray emission followed by a long-lived afterglow phase. The afterglows of GRBs are believed to originate from the synchrotron emission of shock-accelerated electrons produced by the interaction between the outflow and the external medium (Rees \& Mészáros 1992; Mészáros \& Rees 1993, 1997; Sari et al. 1998; Chevalier \& Li 2000). Particle acceleration is usually attributed to the Fermi process (Fermi 1954), which results in a power-law (PL) energy distribution $N(E) \mathrm{d} E \propto E^{-p} \mathrm{~d} E$, with a cutoff at high energies. Some analytical and numerical studies indicate a nearly universal spectral index of $p \sim$ 2.2 - 2.4 (e.g., Bednarz \& Ostrowski 1998; Kirk et al. 2000; Achterberg et al. 2001; Lemoine \& Pelletier 2003; Spitkovsky 2008), though other studies suggest that there is a large range of possible values for $p$ of $1.5-4$ (Baring 2004). The values of $p$ derived from the spectral analysis of the multi-band afterglow (e.g. Chevalier \& Li 2000; Panaitescu \& Kumar 2002; Starling et al. 2008; Curran et al. 2009; Fong et al. 2015; Li et al. 2015; Wang et al. 2015) or the X-ray data alone (e.g., Shen et al. 2006; Curran et al. 2010) show a rather wide distribution, but most of them are consistent with $p>2$. Only a few GRBs, e.g., GRB 060908 (Covino et al. 2010), GRB 091127 (Filgas et al. 2011; Troja et al. 2012), GRB 110918A (Frederiski et al. 2013) and GRB 140515A (Melandri et al. 2015), show very flat spectra in the optical band and require a hard $(p<2)$ electron energy spectrum.

To explain those afterglows that cannot be well modeled with a standard ( $p \gtrsim 2$ ) electron energy spectrum, two types of electron energy distributions were proposed in literature: (1) a single PL electron energy distribution $(1<p<2)$ with an exponential cutoff at a maximum electron Lorentz factor $\gamma_{M}$ (Bhattacharya 2001; Dai \& Cheng 2001); (2) a double PL electron energy distribution $\left(1<p_{1}<2\right.$ and $\left.p_{2}>2\right)$ with an "injection break" $\gamma_{\mathrm{b}}$ (Panaitescu \& Kumar 2001; Bhattacharya \& Resmi 2004; Resmi \& Bhattacharya 2008; Wang et al. 2012). A direct method to distinguish the two models is to see the passage of the injection break frequency $\nu_{\mathrm{b}}$ (i.e., the synchrotron frequency corresponding to $\gamma_{\mathrm{b}}$ ) through a certain band, e.g, from the optical to the near-infrared (NIR) bands. Our previous work (Zhang et al. 2015, Paper I hereafter) showed that GRB 091127 was such a case and gave strong evidence for the double PL hard electron spectrum model (the so-called "DPLH model" in Paper I). The physical origin of $\gamma_{\mathrm{b}}$ is not clear. The DPLH model assumes $\gamma_{\mathrm{b}} \propto \gamma^{q}$ in the highly relativistic regime, here $\gamma$ is the bulk Lorentz factor of the jet. Paper I found $q \sim 0.6$ by modeling the multi-band afterglow of GRB 091127. Does this imply a universal evolution of the injection break? More GRB 091127-like bursts are needed to test this conjecture.

The "smoking-gun" evidence for a DPLH spectrum requires high-quality and multi-wavelength afterglow observations to provide detailed spectral information, in order to identify the existence of $\gamma_{\mathrm{b}}$ and its evolution behavior. In this paper, we show that the multi-band afterglows of GRB 060614 and GRB 060908 can be well modeled by the DPLH model, thus providing further evidence for such a DPLH spectrum. Moreover, the two bursts, along with GRB 091127, seem to show a similar behavior in the evolution of the injection break.

Our paper is organized as follows. In Section 2, we summarize the observational results of GRB 060614 and GRB 060908. Based on the work of Resmi \& Bhattacharya (2008, RB08 hereafter), the DPLH model for both a homogeneous interstellar medium (ISM) and a wind-like circumburst environment is described in Section 3. In this section we also extend the original model by taking into account a continuous energy injection process (Sari \& Mészáros 2000; Zhang \& Mészáros 2001) to explain the afterglow of GRB 060614. In Section 4, we constrain the model parameters and then compare our model with the multi-band afterglow data. Finally, we present our conclusion and make some discussions in Section 5. The convention $F_{\nu} \propto \nu^{-\beta} t^{-\alpha}$ is adopted throughout the paper, where $\beta$ is the spectral index and $\alpha$ is the temporal decay index. We use the standard notation $Q_{x}=Q / 10^{x}$ with $Q$ being a generic quantity in cgs units and assume a concordance cosmology with $H_{0}=70 \mathrm{~km} \mathrm{~s}^{-1} \mathrm{Mpc}^{-1}, \Omega_{\mathrm{M}}=0.27$ and $\Omega_{\Lambda}=0.73$ (Jarosik et al. 2011). All the quoted errors are given at a $1 \sigma$ confidence level (CL) unless stated otherwise.

\section{OBSERVATIONAL RESULTS}

\subsection{GRB 060614}

GRB 060614 triggered the Swift Burst Alert Telescope (BAT; Barthelmy et al. 2005) on 2006 June 14 at $T_{0}=12: 43: 48$ UT (Parsons et al. 2006) and was also detected by Konus-Wind (Golenetskii et al. 2006). The light curve (LC) shows an initial hard, bright peak lasting $\sim 5$ s followed by a long, somewhat softer extended emission, with a total duration of $T_{90}(15-$ $350 \mathrm{keV})=102 \pm 3 \mathrm{~s}$ (Barthelmy et al. 2006). The spectrum of the initial pulse can be fitted in the $20 \mathrm{keV}-$ $2 \mathrm{MeV}$ energy range by a PL with an exponential cutoff model, with the peak energy $E_{\mathrm{pk}} \sim 302 \mathrm{keV}$, while the spectrum of the remaining part of the burst 
can be described by a simple PL with photon index $2.13 \pm 0.03$ (Golenetskii et al. 2006). The total fluence in the $20 \mathrm{keV}-2 \mathrm{MeV}$ energy range is $\sim 4.1 \times 10^{-5}$ $\mathrm{erg} \mathrm{cm}^{-2}$, of which the initial intense pulse contributes a fraction of $\sim 20 \%$ (Golenetskii et al. 2006). With a redshift of $z=0.125$ (Fugazza et al. 2006; Price et al. 2006), the isotropic equivalent energy was estimated as $E_{\gamma, \text { iso }}=(2.5 \pm 0.4) \times 10^{51} \mathrm{erg}$ in the $1-10^{4} \mathrm{keV}$ rest-frame energy band (Mangano et al. 2007, M07 hereafter). In addition, GRB 060614 has null spectral lags, being consistent with typical short GRBs (Gehrels et al. 2006).

The X-ray Telescope (XRT; Burrows et al. 2005) began observing the field $91 \mathrm{~s}$ after the BAT trigger (Parsons et al. 2006). The X-ray afterglow of GRB 060614 exhibits a canonical LC which has been commonly observed in the Swift era (e.g., Nousek et al. 2006; Zhang et al. 2006; Evans et al. 2009). It begins with an initial fast exponential decay, followed by a plateau with slope $\alpha_{\mathrm{X}, 1}=0.11 \pm 0.03$; at $T_{\mathrm{X}, \mathrm{b} 1}=36.6 \pm 1.5 \mathrm{ks}$, it steepens to a standard afterglow evolution with slope $\alpha_{\mathrm{X}, 2}=1.03 \pm 0.01$; later on, the LC shows a further steepening to a slope $\alpha_{\mathrm{X}, 3}=2.13 \pm 0.04$ at $T_{\mathrm{X}, \mathrm{b} 2}=104 \pm 13 \mathrm{ks}$ (M07, see Figure 1$)$. The X-ray data observed in the photon counting (PC) mode show no significant spectral evolution, with the spectral index $\beta_{\mathrm{X}} \sim 0.8(\mathrm{M} 07)$.

The Swift Ultra-Violet/Optical Telescope (UVOT; Roming et al. 2005) commenced observations $101 \mathrm{~s}$ after the BAT trigger (Holland 2006). Besides, the R-band afterglow was detected by several ground telescopes (e.g., Della Valle et al. 2006; French et al. 2006; Fynbo et al. 2006; Gal-Yam et al. 2006). M07 presented detailed spectral and temporal analysis of the optical/ultraviolet (UV) afterglow, below we summarize their main results. The optical/UV LCs show achromatic breaks with the X-ray afterglow, i.e., $t_{\mathrm{UVO}, \mathrm{b} 1}=29.7 \pm 2.7 \mathrm{ks}$ and $t_{\mathrm{UVO}, \mathrm{b} 2}=117.2 \pm 2.7 \mathrm{ks}$. The decay slopes after the two breaks are $\alpha_{\mathrm{UVO}, 2}=1.11 \pm 0.03$ and $\alpha_{\mathrm{UVO}, 3}=2.44 \pm 0.05$, respectively. On the whole, the X-ray/UV/optical LCs have marginally consistent evolutions after $\sim 30 \mathrm{ks}$. What is puzzling is that the initial slope $\alpha_{\mathrm{UVO}, 1}$ is dependent on wavelength: the UV LCs show nearly flat evolutions while the optical LCs rise slowly with slopes from $\sim(-0.38)$ to $\sim(-0.17)$ (see Figure 1). The spectral energy distributions (SEDs) of the afterglow from optical to X-rays show a spectral break passing through the optical/UV band between $\sim 10$ and $\sim 30 \mathrm{ks}$. The break frequency at $10 \mathrm{ks}$ is around $1.0 \times 10^{15} \mathrm{~Hz}$ (see Figure 7 of M07). At this time, the optical/UV and X-ray afterglows have spectral indices $\beta_{\mathrm{UVO}}=0.30 \pm 0.09$ and $\beta_{\mathrm{X}}=0.84 \pm 0.04$, respectively. At later times ( $t \gtrsim 30 \mathrm{ks}$ ), the spectral index in the optical/UV band changes to be consistent with that of X-rays. Fits of the broad-band SEDs imply a weak host extinction $A_{V, \mathrm{~h}}=0.05 \pm 0.01$ (M07).

In addition, deep optical/NIR follow-ups of GRB 060614 show no evidence for an associated supernova down to very strict limits; the GRB host is a very faint starforming galaxy with a specific star formation rate lower than most long GRB hosts; the GRB counterpart resides in the outskirts of the host (Della Valle et al. 2006; Fynbo et al. 2006; Gal-Yam et al. 2006). The recent discovery of a distinct NIR excess at about 13.6 days after the burst suggests a possible kilonova (or macronova) origin (Jin et al. 2015; Yang et al. 2015). Together with the vanishing time lags of the prompt emission, all these point towards a different origin from typical long GRBs; it is likely to be of a subclass of merger-type short GRBs (Gehrels et al. 2006; Zhang et al. 2007).

\subsection{GRB 060908}

GRB 060908 triggered the Swift/BAT on 2006 September 14 at $T_{\mathrm{BAT}}=08: 57: 22.34 \mathrm{UT}$ (Evans et al. 2006). Further analysis found the onset of the GRB occurs $12.96 \mathrm{~s}$ before the trigger time, i.e., $T_{0}=$ $T_{\mathrm{BAT}}-12.96 \mathrm{~s}$ (Covino et al. 2010). So the time used in this work is relative to $T_{0}$. The BAT LC shows a multi-peaked structure with a total duration of $T_{90}(15-350 \mathrm{keV})=19.3 \pm 0.2 \mathrm{~s}$ (Palmer et al. 2006). The time-averaged spectrum is best fit by a simple PL and can be alternatively fit by a Band function (Band et al. 1993) with the high-energy photon index fixed. With a redshift of $z=1.884$ (Fynbo et al. 2009), the latter spectral model gives the rest-frame peak energy $E_{\mathrm{p}, \mathrm{i}} \sim 380 \mathrm{keV}$ and the isotropic equivalent energy $E_{\gamma, \text { iso }}=(6.2 \pm 0.4) \times 10^{52}$ erg in the rest-frame $1-10^{4} \mathrm{keV}$ energy band (Covino et al. 2010).

The XRT began observing the field $72 \mathrm{~s}$ after the BAT trigger(Evans et al. 2006). The spectra were modeled with an absorbed power-law, which gave the spectral index $\beta_{\mathrm{X}}=1.17_{-0.22}^{+0.25}$ and the host absorbing column density $N_{\mathrm{H}} \sim 8.3 \times 10^{21} \mathrm{~cm}^{-2}$. The LC is characterised by a constant PL decay with index $\alpha_{\mathrm{X}}=1.12_{-0.02}^{+0.05}$, while from $\sim 200$ to $\sim 1000 \mathrm{~s}$ a complex flaring activity is superposed on the underlying decay (Covino et al. 2010, see Figure 2).

The UVOT commenced observations $80 \mathrm{~s}$ after the BAT trigger (Morgan et al. 2006). The optical/NIR afterglow was also monitored by several ground-based telescopes (e.g., Andreev et al. 2006; Antonelli et al. 2006; Nysewander et al. 2006; Wiersema et al. 2006). The LCs can be described by a broken PL with the initial decay index $\alpha_{\text {optNIR,1 }}=1.48 \pm 0.25$, the break time 
$t_{\mathrm{optNIR}, 1}=138_{-43}^{+167} \mathrm{~s}$ and the post-break decay index $\alpha_{\text {optNIR }, 2}=1.05 \pm 0.03$ (Covino et al. 2010). There seems to be another break at $\sim 10^{3}-10^{4} \mathrm{~s}$, with the post-break decay slope of $1.1-1.4$ (see Figure 2). However, this break time cannot be well constrained by the data (Covino et al. 2010). In Subsection 4.2, we will show that such a late break is actually required by the afterglow modeling. The spectral analysis at 800 and $8000 \mathrm{~s}$ shows rather flat spectra with index $\beta_{\mathrm{optNIR}}=$ $0.33_{-0.29}^{+0.25}$ and host dust extinction $E(B-V) \sim 0.03$ (Covino et al. 2010).

\section{MODEL}

Several clues should be considered before establishing the afterglow model for both GRBs. For GRB 060614: (i) The two achromatic breaks $\left(t_{\mathrm{b}, 1} \equiv t_{\mathrm{UVO}, \mathrm{b} 1} \approx t_{\mathrm{X}, \mathrm{b} 1}\right.$ and $\left.t_{\mathrm{b}, 2} \equiv t_{\mathrm{UVO}, \mathrm{b} 2} \approx t_{\mathrm{X}, \mathrm{b} 2}\right)$ shown in the multi-band LCs require a hydrodynamical origin. This canonical afterglow behavior was well described in Zhang et al. (2006). The first break is possibly an "energy-injection break", implying the end of a continuous energy injection into the forward shock (Sari \& Mészáros 2000; Zhang \& Mészáros 2001), while the second break is most likely the so-called "jet break" (Rhoads 1999; Sari et al. 1999); (ii) The early flat spectrum ( $\left.\beta_{\mathrm{UVO}} \sim 0.3\right)$ in the optical/UV band definitely requires a hard electron energy spectrum; (iii) There should be a spectral break between the optical/UV and the X-ray bands, but neither the minimum synchrotron frequency $\nu_{\mathrm{m}}$ nor the cooling frequency $\nu_{\mathrm{c}}$ can accommodate the observations ${ }^{1}$. For GRB 060908, the SED analysis also requires a hard electron energy distribution and some kind of spectral break between the optical/NIR and the X-ray bands. The single PL hard electron spectrum model of Dai \& Cheng (2001) with $\nu_{\mathrm{optNIR}}<\nu_{\mathrm{c}}<\nu_{\mathrm{X}}$ has difficulties in explaining the observations, since the model predicts $\beta_{\mathrm{X}} \sim 0.8$ which is obviously lower than the observed value; the predicted decay slopes are also inconsistent with the observations. Therefore, the DPLH model is a natural choice.

Using the derived spectral and temporal indices of RB08 (their Table 2), we found the afterglow properties of GRB 060614 can be well reproduced by the DPLH

\footnotetext{
${ }^{1}$ The reasons are as follows: (i) Although the passage of $\nu_{\mathrm{m}}$ can produce a spectral evolution and slow-rising optical LCs (M07), this requires $\nu_{\mathrm{opt}}<\nu_{\mathrm{m}}$. The model predicted spectral index in this regime is $\beta_{\mathrm{opt}}=-1 / 3$ which is inconsistent with the observed value $(\sim 0.3)$; (ii) If the observed break frequency is $\nu_{\mathrm{c}}$, it suggests a hard electron energy spectrum with $p=2 \beta_{\text {UVO }}+1 \sim 1.6$. However, the single PL hard electron spectrum model of Dai \& Cheng (2001) predicts the post-jet-break decay slope should be $\sim 1.9$ which is substantially lower than the observed value $(\sim 2.4 ; \mathrm{M} 07)$.
}

model for an ISM medium when an additional energy injection is invoked, while the properties of GRB 060908 can be well explained when a wind-like circumburst density profile is used. In this section, we give a basic description of the DPLH model and present relevant formulas which will be used in Section 4. We refer the reader to $\mathrm{RB} 08$ for more details.

The DPLH spectrum with indices $1<p_{1}<2$ and $p_{2}>2$ is represented as (RB08)

$$
N\left(\gamma_{\mathrm{e}}\right)=C_{\mathrm{e}}\left\{\begin{array}{l}
\left(\frac{\gamma_{\mathrm{e}}}{\gamma_{\mathrm{b}}}\right)^{-p_{1}}, \gamma_{\mathrm{m}} \leqslant \gamma_{\mathrm{e}}<\gamma_{\mathrm{b}}, \\
\left(\frac{\gamma_{\mathrm{e}}}{\gamma_{\mathrm{b}}}\right)^{-p_{2}}, \gamma_{\mathrm{e}} \geqslant \gamma_{\mathrm{b}}
\end{array}\right.
$$

where $C_{\mathrm{e}}$ is the normalization constant, $\gamma_{\mathrm{m}}$ is minimum electron Lorentz factors, and $\gamma_{\mathrm{b}}$ is the injection break. The physical origin of $\gamma_{\mathbf{b}}$ is not clear, RB08 assumed that it is a function of $\beta \gamma$ to accommodate the nonrelativistic regime of expansion, i.e.,

$$
\gamma_{\mathrm{b}}=\xi(\beta \gamma)^{q}
$$

where $\xi$ is a constant of proportionality, $\beta=\sqrt{1-\gamma^{-2}}$ is the dimensionless bulk velocity, and $q$ is assumed to be a constant for simplicity.

For a a relativistic shock propagating through a cold medium with particle density $n$, the post-shock particle density and energy density are $4 \gamma n$ and $4 \gamma(\gamma-1) n m_{\mathrm{p}} c^{2}$, respectively (Sari et al. 1998), from which one derives the minimum Lorentz factor (RB08)

$\gamma_{\mathrm{m}}=\left(f_{\mathrm{p}} \frac{m_{\mathrm{p}}}{m_{\mathrm{e}}} \frac{\epsilon_{\mathrm{e}}}{\xi^{2-p_{1}}}\right)^{\frac{1}{p_{1}-1}} \beta^{-\frac{q\left(2-p_{1}\right)}{p_{1}-1}}(\gamma-1)^{\frac{1}{p_{1}-1}} \gamma^{-\frac{q\left(2-p_{1}\right)}{p_{1}-1}}$

where $m_{\mathrm{p}}$ and $m_{\mathrm{e}}$ are the proton and electron rest mass, respectively; $\epsilon_{\mathrm{e}}$ is the fraction of shock energy carried by electrons, and $f_{\mathrm{p}}=\left[\left(2-p_{1}\right)\left(p_{2}-2\right)\right] /\left[\left(p_{1}-1\right)\left(p_{2}-p_{1}\right)\right]$.

We calculate the break frequencies of synchrotron spectra $\nu_{\mathrm{m}}, \nu_{\mathrm{b}}, \nu_{\mathrm{c}}$ and the peak flux $F_{\nu, \text { max }}$ according to the formulas given by Wijers \& Galama (1999):

$$
\begin{aligned}
\nu_{\mathrm{m}} & =\frac{x_{p}}{1+z} \frac{q_{\mathrm{e}} B^{\prime}}{\pi m_{\mathrm{e}} c} \gamma \gamma_{\mathrm{m}}^{2}, \\
\nu_{\mathrm{b}, \mathrm{c}} & =\frac{0.286}{1+z} \frac{q_{\mathrm{e}} B^{\prime}}{\pi m_{\mathrm{e}} c} \gamma \gamma_{\mathrm{b}, \mathrm{c}}^{2}, \\
F_{\nu, \max } & =\frac{\sqrt{3} \phi_{p} N_{\mathrm{e}} q_{\mathrm{e}}^{3}(1+z)}{4 \pi d_{\mathrm{L}}^{2} m_{\mathrm{e}} c^{2}} B^{\prime} \gamma
\end{aligned}
$$

where $N_{\mathrm{e}}$ is the total number of swept-up electrons, $q_{\mathrm{e}}$ is the electron charge, $B^{\prime}=\left(32 \pi n m_{\mathrm{p}} c^{2} \epsilon_{\mathrm{B}}\right)^{1 / 2} \gamma$ is the post-shock magnetic field density, $\epsilon_{\mathrm{B}}$ is the fraction of shock energy carried by magnetic fields, $d_{\mathrm{L}}$ is the luminosity distance corresponding to the redshift 
$z, \gamma_{\mathrm{c}}=6 \pi m_{\mathrm{e}} c /\left(\sigma_{\mathrm{T}} \gamma B^{\prime 2} t\right)$ is the cooling Lorentz factor of electrons. $x_{p}$ and $\phi_{p}$ represent the dimensionless peak frequency and the peak flux, respectively. Their dependence on $p$ can be obtained from Wijers \& Galama (1999).

For the adiabatic self-similar evolution of a spherical blastwave, the radius $r$ and bulk Lorentz factor $\gamma$ evolve as $r=\left[(17-4 k)(4-k) E t / 4 \pi A m_{\mathrm{p}} c(1+\right.$ $z)]^{1 /(4-k)}$ and $\gamma=\left[(17-4 k) E(1+z)^{3-k} / 4^{5-k}(4-\right.$ $\left.k)^{3-k} \pi A m_{\mathrm{p}} c^{5-k} t^{3-k}\right]^{1 / 2(4-k)}$ in the ultra-relativistic regime (Blandford \& McKee 1976; Sari et al. 1998; Chevalier \& Li 2000; Gao et al. 2013). The above derivation used the density profile $n=A r^{-k}, k=0$ for ISM and $k=2$ for wind medium. By substituting these expressions in Equations (4)-(6), one derives ${ }^{2}$

$$
\begin{aligned}
\nu_{\mathrm{m}}= & 8.2 \times 10^{6}\left(1833 f_{\mathrm{p}}\right)^{\frac{2}{p_{1}-1}}(37.2)^{\frac{1-q\left(2-p_{1}\right)}{p_{1}-1}} \frac{x_{p_{1}}}{1+z} \\
& \xi^{\frac{-2\left(2-p_{1}\right)}{p_{1}-1}} \epsilon_{\mathrm{e}}^{\frac{2}{p_{1}-1}} \epsilon_{\mathrm{B},-2}^{1 / 2} E_{52}^{\frac{p_{1}-q\left(2-p_{1}\right)}{4\left(p_{1}-1\right)}} n_{0}^{\frac{p_{1}-2+q\left(2-p_{1}\right)}{4}} \\
& \left(\frac{t_{\mathrm{d}}}{1+z}\right)^{\frac{-3\left[p_{1}-q\left(2-p_{1}\right)\right]}{4\left(p_{1}-1\right)}} \mathrm{Hz}, \\
\nu_{\mathrm{c}}= & \left.1.5 \times 10^{15} \epsilon_{\mathrm{B},-2}^{-3 / 2} E_{52}^{-1 / 2} n_{0}^{-1}\left[t_{\mathrm{d}}(1+z)\right]^{-1 / 2} \mathrm{H} \not, 8\right) \\
\nu_{\mathrm{b}}= & 3.8 \times 10^{5} \frac{(6.1)^{1+2 q}}{1+z} \xi^{2} \epsilon_{\mathrm{B},-2}^{1 / 2} E_{52}^{\frac{1+q}{4}} n_{0}^{\frac{1-q}{4}} \\
& \left(\frac{t_{\mathrm{d}}}{1+z}\right)^{-\frac{3(1+q)}{4}} \mathrm{~Hz}, \\
F_{\nu, \max }= & 6.8 \times 10^{3} \phi_{p_{1}} \epsilon_{\mathrm{B},-2}^{1 / 2} E_{52} n_{0}^{1 / 2} d_{\mathrm{L}, 28}^{-2}(1+z) \mu \mathrm{Jy}(10)
\end{aligned}
$$

for the ISM case, and

$$
\begin{aligned}
\nu_{\mathrm{m}}= & 5.8 \times 10^{6}(13.8)^{y}\left(183.3 f_{\mathrm{p}}\right)^{\frac{2}{p_{1}-1}} \frac{x_{p_{1}}}{1+z} \xi^{\frac{-2\left(2-p_{1}\right)}{p_{1}-1}} \\
& \epsilon_{\mathrm{e},-1}^{\frac{2}{p_{1}-1}} \epsilon_{\mathrm{B},-2}^{1 / 2} E_{52}^{y / 2} A_{*}^{\frac{1-y}{2}}\left(\frac{t_{\mathrm{d}}}{1+z}\right)^{-\frac{2+y}{2}} \mathrm{~Hz}, \quad(11) \\
\nu_{\mathrm{c}}= & \frac{1.6 \times 10^{15}}{(1+z)^{3}} \epsilon_{\mathrm{B},-2}^{-3 / 2} E_{52}^{1 / 2} A_{*}^{-2}\left(\frac{t_{\mathrm{d}}}{1+z}\right)^{1 / 2} \mathrm{~Hz},(12 \\
\nu_{\mathrm{b}}= & 1.6 \times 10^{6} \frac{(13.8)^{q}}{1+z} \xi^{2} \epsilon_{\mathrm{B},-2}^{1 / 2} E_{52}^{q / 2} A_{*}^{\frac{1-q}{2}} \\
& \left(\frac{t_{\mathrm{d}}}{1+z}\right)^{-\frac{2+q}{2}} \mathrm{~Hz}, \\
F_{\nu, \max }= & 20.6 \phi_{p_{1}}(1+z) \epsilon_{\mathrm{B},-2}^{1 / 2} E_{52}^{1 / 2} A_{*} d_{\mathrm{L}, 28}^{-2}
\end{aligned}
$$

\footnotetext{
2 Different from RB08, we did not consider the effect of sideways expansion in the derivation of Equations (7)-(14). This can be seen as a reasonable approximate in the ultra-relativistic regime as long as the inverse Lorentz factor has not exceeded the initial jet opening angle (Rhoads 1999). The coefficients in these equations are consistent with those of RB08 within a factor of a few that may be due to minor differences in the treatment of dynamics.
}

$$
\left(\frac{t_{\mathrm{d}}}{1+z}\right)^{-1 / 2} \mathrm{mJy}
$$

for the wind case $^{3}$

The evolution of the synchrotron flux density at a given frequency $\left(F_{\nu}\right)$ relies on the order of the three break frequencies and the regime in which $\nu$ resides. Below we give only some scaling laws ${ }^{4}$ for $F_{\nu}$ and $\nu_{\mathrm{b}}$ that will be used in Section 4. Since the synchrotron selfabsorption process is not relevant, we do not consider it in this work.

For GRB 060908, according to Equations (11)-(14), the relevant spectral regimes and flux densities are:

(i) $\nu_{\mathrm{m}}<\nu<\min \left(\nu_{\mathrm{b}}, \nu_{\mathrm{c}}\right)$

$$
F_{\nu}=F_{\nu, \max }\left(\frac{\nu}{\nu_{\mathrm{m}}}\right)^{-\frac{p_{1}-1}{2}} \propto t^{\frac{1}{4}\left(2 q-p_{1} q-2 p_{1}-1\right)} .
$$

(ii) $\nu_{\mathrm{m}}<\nu_{\mathrm{b}}<\nu<\nu_{\mathrm{c}}$

$$
\begin{aligned}
F_{\nu} & =F_{\nu, \max }\left(\frac{\nu_{\mathrm{b}}}{\nu_{\mathrm{m}}}\right)^{-\frac{p_{1}-1}{2}}\left(\frac{\nu}{\nu_{\mathrm{b}}}\right)^{-\frac{p_{2}-1}{2}} \\
& \propto t^{\frac{1}{4}\left(2 q-p_{2} q-2 p_{2}-1\right)}
\end{aligned}
$$

(iii) $\nu>\max \left(\nu_{\mathrm{b}}, \nu_{\mathrm{c}}\right)>\nu_{\mathrm{m}}$,

$$
\begin{aligned}
F_{\nu} & =F_{\nu, \max }\left(\frac{\nu_{\mathrm{c}}}{\nu_{\mathrm{m}}}\right)^{-\frac{p_{1}-1}{2}}\left(\frac{\nu_{\mathrm{b}}}{\nu_{\mathrm{c}}}\right)^{-\frac{p_{1}}{2}}\left(\frac{\nu}{\nu_{\mathrm{b}}}\right)^{-\frac{p_{2}}{2}} \\
& =F_{\nu, \max }\left(\frac{\nu_{\mathrm{b}}}{\nu_{\mathrm{m}}}\right)^{-\frac{p_{1}-1}{2}}\left(\frac{\nu_{\mathrm{c}}}{\nu_{\mathrm{b}}}\right)^{-\frac{p_{2}-1}{2}}\left(\frac{\nu}{\nu_{\mathrm{c}}}\right)^{-\frac{p_{2}}{2}} \\
& \propto t^{\frac{1}{4}\left(2 q-p_{2} q-2 p_{2}\right)} .
\end{aligned}
$$

For GRB 060614, the situation is somewhat more complicated. Besides the adiabatic self-similar evolution phase, these should be a continuous energy injection process before $\sim 30 \mathrm{ks}$ and a jet break at about $117 \mathrm{ks}$. The injected energy can be provided by a long-lived central engine (Dai \& Lu 1998; Zhang \& Mészáros 2001) or by slower material with significant energy which gradually piles up onto the decelerating ejecta and "refreshes" it (Ress \& Mészáros 1998; Sari \& Mészáros 2000). Here we do not consider a specific energy injection mechanism and generally assume that the isotropic equivalent

\footnotetext{
${ }^{3}$ We note the exponent of $\xi$ in Equation (11) and the exponent of $A_{*}$ in Equation (13) are different from the results of RB08 (see their Equations (13) and (15)). Their expression of $\nu_{\mathrm{b}}$ also missed out a factor of $1 /(1+z)$. We have carefully checked our derivations to make sure that our results are robust. Here $t_{\mathrm{d}}$ is the time in days, $A_{*}$ and $A$ are related by $A=3 \times 10^{35} A_{*} \mathrm{~cm}^{-1}$ and $y=\left[1-q\left(2-p_{1}\right)\right] /\left(p_{1}-1\right)$.

4 We refer the reader to $\mathrm{RB} 08$ for a complete reference of the scaling relationships for the spectral breaks and $F_{\nu}$ in various spectral regimes (but without an energy injection).
} 
blastwave energy $E$ evolves as

$$
E(t)= \begin{cases}E_{\mathrm{f}}\left(\frac{t}{t_{\mathrm{f}}}\right)^{1-e}, & t_{\mathrm{i}} \leqslant t<t_{\mathrm{f}} \\ E_{\mathrm{f}}, & t \geqslant t_{\mathrm{f}}\end{cases}
$$

where $t_{\mathrm{i}}$ is the time when the assumed PL energy injection $\left(E \propto t^{1-e}\right)$ begins, $t_{\mathrm{f}}$ is the end time of the energy injection, $E_{\mathrm{f}}$ is the final blastwave energy, and $e<1$ is required for an effective energy injection. When this energy injection is taken into account, the blastwave energy $E$ in Equations (7)-(10) should be replaced with Equation (18).

According to Equations (7)-(10), the relevant spectral regimes and flux densities are:

(i) $\nu_{\mathrm{m}}<\nu<\nu_{\mathrm{b}}<\nu_{\mathrm{c}}$

$$
F_{\nu}=F_{\nu, \max }\left(\frac{\nu}{\nu_{\mathrm{m}}}\right)^{-\frac{p_{1}-1}{2}} \propto t^{\left[(1-e)-\frac{(2+e)\left(p_{1}+p_{1} q-2 q\right)}{8}\right]} .
$$

(ii) $\nu_{\mathrm{m}}<\nu_{\mathrm{b}}<\nu<\nu_{\mathrm{c}}$,

$$
\begin{aligned}
F_{\nu} & =F_{\nu, \max }\left(\frac{\nu_{\mathrm{b}}}{\nu_{\mathrm{m}}}\right)^{-\frac{p_{1}-1}{2}}\left(\frac{\nu}{\nu_{\mathrm{b}}}\right)^{-\frac{p_{2}-1}{2}} \\
& \propto t^{\left[(1-e)-\frac{(2+e)\left(p_{2}+p_{2} q-2 q\right)}{8}\right]} .
\end{aligned}
$$

According to Equation (9), the injection break frequency $\nu_{\mathrm{b}}$ scales as

$$
\nu_{\mathrm{b}} \propto t^{-\frac{(2+e)(1+q)}{4}} .
$$

After the end of the energy injection $\left(t \geqslant t_{\mathrm{f}}\right)$, the blastwave enters an adiabatic evolution phase and the corresponding scaling relationships can be easily obtained by setting $e=1$ in Equations (19)-(21).

We next discuss the physical origin of the jet break of GRB 060614. For a simplified conical jet with a halfopening angle $\theta_{\mathrm{j}}$, as it decelerates, the radiation beaming angle $(1 / \gamma)$ would eventually exceed the jet half-opening angle, i.e., $1 / \gamma>\theta_{\mathrm{j}}$. At this time, a jet break may occur in the afterglow LC. Two effects could result in a jet break: the first is the pure jet-edge effect which steepens the LC by $t^{-3 / 4}$ for an ISM medium (Mészáros \& Rees 1999); the second effect is caused by sideways expansion, which has important effects on the hydrodynamics when $1 / \gamma \gtrsim \theta_{\mathrm{j}}$ is satisfied and the post-jet-break flux decays as $t^{-p}$ for a normal electron energy spectrum with index $p>2$ (Rhoads 1999; Sari et al. 1999).

For GRB 060614, the jet break should be a result of significant sideways expansion rather than the jet-edge effect. The reasons are as follows: (i) The post-jet-break decay (in the optical/UV band) caused by the edge effect would have a slope $\sim 1.1+0.75=1.85$, which is substantially lower than the observed value $(\sim 2.44)$; (ii) Using the expression (their Equation (11)) given by Wang et al. (2012) who considered the effect of sideways expansion in a similar DPLH model and the obtained parameter values $\left(p_{2}\right.$ and $q$ ) in Subsection 4.1 , we estimate the post-jet-break slope to be $\sim 2.48$ which is excellently consistent with the observed value.

Based on the work of Wang et al. (2012), we give the scaling law for $F_{\nu}$ in the post-jet-break phase straightforwardly. For $\nu_{\mathrm{m}}<\nu_{\mathrm{b}}<\nu<\nu_{\mathrm{c}}$,

$$
F_{\nu} \propto t^{-\frac{q\left(p_{2}-2\right)+\left(p_{2}+2\right)}{2}}, \quad t>t_{\mathrm{j}}
$$

where $t_{\mathrm{j}}$ is the jet-break time.

\section{PARAMETER CONSTRAINT AND AFTERGLOW MODELING}

\subsection{GRB 060614}

Before constraining the free parameters $\left(p_{1}, p_{2}, q, e\right.$, $\epsilon_{\mathrm{e}}, \epsilon_{\mathrm{B}}, \xi, E_{\mathrm{f}}$ and $\left.n\right)$, we first summarize the relevant observational results of GRB 060614: (i) $\beta_{\mathrm{UVO}}(10 \mathrm{ks})=$ $0.30 \pm 0.09, \beta_{\mathrm{X}}=0.84 \pm 0.04$; (ii) $\alpha_{\mathrm{X}, 1}=0.11 \pm 0.03$; $\alpha_{\mathrm{UVO}, 2}=1.11 \pm 0.03 \approx \alpha_{\mathrm{X}, 2} ;$ (iii) $t_{\mathrm{b}, 1}=29.7 \pm 2.7 \mathrm{ks}$, $t_{\mathrm{b}, 2}=117.2 \pm 2.7 \mathrm{ks} ;\left(\right.$ iv) $\tilde{\nu}_{\mathrm{b}}(10 \mathrm{ks}) \approx 1.0 \times 10^{15} \mathrm{~Hz}^{5}$; (v) $\tilde{\nu}_{\mathrm{b}}(30 \mathrm{ks}) \lesssim \nu_{\mathrm{R}}$, since the SED shows that the break frequency has just crossed the R-band at about $30 \mathrm{ks}$; (vi) the initial decay slope of the $R$-band $\mathrm{LC} \alpha_{\mathrm{R}, 1}=$ $-0.38 \pm 0.14$; (vii) $\alpha_{\mathrm{UVO}, 3}=2.44 \pm 0.05 \approx \alpha_{\mathrm{X}, 3}$. In this section we use conditions (i)-(iv) to constrain the model parameters, and use (v)-(vii) for consistency checks.

Using condition (i), we get $p_{1}=2 \beta_{\mathrm{UVO}}(10 \mathrm{ks})+1=$ $1.60 \pm 0.18$ and $p_{2}=2 \beta_{\mathrm{X}}+1=2.68 \pm 0.08$. The values of $q$ and $e$ can be obtained from condition (ii) and Equation (20), i.e.,

$$
\begin{array}{r}
\frac{(2+e)\left(p_{2}+p_{2} q-2 q\right)}{8}-(1-e)=0.11 \pm 0.03 \\
\frac{3\left(p_{2}+p_{2} q-2 q\right)}{8}=1.11 \pm 0.03
\end{array}
$$

Solving these equations gives $q=0.41 \pm 0.20$ and $e=0.27 \pm 0.04$. With these values, we test our model predictions with conditions (v)-(vii). First, Equation (21) gives $\nu_{\mathrm{b}} \propto t^{-0.80 \pm 0.12}$ during the energy injection phase, then, with condition (iv) we have $\nu_{\mathrm{b}}(30 \mathrm{ks}) \approx$ $4.2 \times 10^{14} \mathrm{~Hz}$, which is excellently consistent with condition (v). Second, based on Equation (19), the predicted initial $R$-band decay slope is $-0.32 \pm 0.09$ that is consistent with the observational results (condition

\footnotetext{
${ }^{5}$ Here and below we use $\tilde{\nu}_{\mathrm{b}}$ to denote the observed break frequency in the SEDs, in order to distinguish with the injection break frequency $\nu_{\mathrm{b}}$ in our model.
} 
(vi)) within $1 \sigma$ errors. Finally, we estimate the postjet-break decay slope from Equation (22) and the obtained value is $2.48 \pm 0.09$, which is in perfect accord with that of the optical/UV afterglow, and marginally consistent with that of the X-ray afterglow. These exciting results encourage us to have a further check of our model by modeling the afterglow LCs. In the following calculations, we adopt $p_{1}=1.6, p_{2}=2.68, q=0.41$ and $e=0.27$.

In the normal decay phase $\left(t_{\mathrm{b}, 1}<t<t_{\mathrm{b}, 2}\right)$, we have $\nu_{\mathrm{m}}<\nu_{\mathrm{b}}<\nu_{\mathrm{UVO}}<\nu_{\mathrm{X}}<\nu_{\mathrm{c}}$. Following Equations (7)(10) and (20), one derives ${ }^{6}$

$$
\begin{aligned}
\nu_{\mathrm{m}} & \left.=1.1 \times 10^{10} \xi_{4}^{-1.33} \epsilon_{\mathrm{e},-1}^{3.33} \epsilon_{\mathrm{B},-2}^{1 / 2} E_{\mathrm{f}, 52}^{0.6} n_{0}^{-0.06} t_{\mathrm{d}}^{-1.8} \mathrm{H} \notin 25\right) \\
\nu_{\mathrm{b}} & =1.0 \times 10^{15} \xi_{4}^{2} \epsilon_{\mathrm{B},-2}^{1 / 2} E_{\mathrm{f}, 52}^{0.35} n_{0}^{0.15} t_{\mathrm{d}}^{-1.06} \mathrm{~Hz}, \\
\nu_{\mathrm{c}} & =1.4 \times 10^{15} \epsilon_{\mathrm{B},-2}^{-3 / 2} E_{\mathrm{f}, 52}^{-1 / 2} n_{0}^{-1} t_{\mathrm{d}}^{-1 / 2} \mathrm{~Hz}, \\
F_{\nu_{\mathrm{R}}} & =7.5 \times 10^{3} \xi_{4}^{0.68} \epsilon_{\mathrm{e},-1} \epsilon_{\mathrm{B},-2}^{0.92} E_{\mathrm{f}, 52}^{1.37} n_{0}^{0.56} t_{\mathrm{d}}^{-1.11} \mu \mathrm{Jy} .(28)
\end{aligned}
$$

To constrain the parameters, we require that (i) the $R$-band flux at $52 \mathrm{ks}$ is $F_{\nu_{\mathrm{R}}}(52 \mathrm{ks})=55.9 \mu \mathrm{Jy}^{7}$, (ii) $\nu_{\mathrm{b}}(10 \mathrm{ks})=1.0 \times 10^{15} \mathrm{~Hz}$, and (iii) $\nu_{\mathrm{c}}$ should well above $10 \mathrm{keV}$ at the last measurement of the X-ray afterglow, i.e., $\nu_{\mathrm{c}}\left(2 \times 10^{6} \mathrm{~s}\right)>10 \mathrm{keV}$. After a simple calculation, we get

$$
\begin{aligned}
\epsilon_{\mathrm{B},-2} n_{0}^{2 / 3} & =1.95 \times 10^{-3} \epsilon_{\mathrm{e},-1}^{-4 / 3} E_{\mathrm{f}, 52}^{-5 / 3}, \\
\xi_{4} & =1.5 \epsilon_{\mathrm{e},-1}^{-1 / 3} E_{\mathrm{f}, 52}^{0.24} n_{0}^{0.09}, \\
\epsilon_{\mathrm{e},-1} & >0.84 E_{\mathrm{f}, 52}^{-1} .
\end{aligned}
$$

With only two equations, the model parameters $\left(\epsilon_{\mathrm{e}}\right.$, $\epsilon_{\mathrm{B}}, \xi, E_{\mathrm{f}}$ and $\left.n\right)$ are strongly degenerate. Here we adopt a typical value of $\epsilon_{\mathrm{e},-1}=1$, which has been supported by recent large sample afterglow modelings (e.g., Nava et al. 2014; Santana et al. 2014; Beniamini \& van der Horst 2017). $E_{\mathrm{f}}$ is the final blastwave energy after the energy injection, of which the mechanism was not specified above. Here we simply assume an equivalent prompt emission efficiency of $\eta_{\gamma}=E_{\gamma} /\left(E_{\gamma}+E_{\mathrm{f}}\right)=10 \%$ and leave the discussion on the energy injection mechanism in Section 5. With $E_{\gamma}=2.5 \times 10^{51} \mathrm{erg}$ and $\eta_{\gamma}=10 \%$, we obtain $E_{\mathrm{f}, 52}=2.25$ and Equation (31) is naturally satisfied. By substituting these values in Equation (29), we get

\footnotetext{
${ }^{6} x_{p_{1}}=0.85$ and $\phi_{p_{1}}=0.5$ were adopted in the derivations according to Wijers \& Galama (1999) and our obtained $p_{1}=1.6$. The same values were used to calculate $\nu_{\mathrm{m}}$ and $F_{\nu, \max }$ for GRB 060908 in Subsection 4.2.

7 This value has been corrected for Galactic and host galaxy extinction with $A_{V, \mathrm{G}}=0.07$ and $A_{V, \mathrm{~h}}=0.05$, respectively, according to the results of M07.
}

$\epsilon_{\mathrm{B},-2} n_{0}^{2 / 3}=5.0 \times 10^{-4}$. The values of $\epsilon_{\mathrm{B}}$ and $n$ cannot be well constrained since both of them are highly uncertain parameters and vary over several orders of magnitude. By modeling the multi-band afterglows of 38 short GRBs, Fong et al. (2015) gave a median density of $n \sim 10^{-3}-10^{-2} \mathrm{~cm}^{-3}$, and found that $80 \%-95 \%$ of bursts have densities of $n \lesssim 1 \mathrm{~cm}^{-3}$. For GRB 060614, if we take $n_{0}=10^{-1}$ to $10^{-3}$, we get $\epsilon_{\mathrm{B},-2}=2.3 \times 10^{-3}$ to $5 \times 10^{-2}$. These values are well consistent with the recent results of Santana et al. (2014) and Barniol Duran (2014), who found the distribution of $\epsilon_{\mathrm{B}}$ has a range of $\sim 10^{-8}-10^{-3}$ with a median value of $\sim$ a few $\times 10^{-5}$. In the following calculations, we adopt $n_{0}=0.01$ and $\epsilon_{\mathrm{B},-2}=1.1 \times 10^{-2}$. Finally, we substitute the above values in Equation (30) and get $\xi_{4}=1.2$. We note $\xi$ is weakly dependent on other parameters and can be well constrained; it is around $10^{4}$, varying within a factor of two.

Since we interpret the achromatic break at $t_{\mathrm{b}, 2}$ as a jet break, we can estimate the half-opening angle of the jet according to $\theta_{\mathrm{j}} \sim \gamma\left(t_{\mathrm{j}}\right)^{-1}$ (Rhoads 1999; Sari et al. 1999). We thus have

$$
\theta_{\mathrm{j}}=9.4^{\circ} E_{52}^{-1 / 8} n_{0}^{1 / 8}\left(\frac{t_{\mathrm{b}, 2, \mathrm{~d}}}{1+z}\right)^{3 / 8}=5.1^{\circ} .
$$

Using $\gamma\left(t_{\mathrm{j}}\right) \sim \theta_{\mathrm{j}}^{-1}=11.2$ and $\gamma\left(t>t_{\mathrm{j}}\right) \propto t^{-1 / 2}$ (Rhoads 1999), we have $\gamma\left(2 \times 10^{6} \mathrm{~s}\right) \sim 2.7$, which suggests a mildly relativistic jet even at the end of the X-ray observations. Therefore, our explanation of the entire afterglow of GRB 060614 in the highly relativistic regime is self-consistent.

Based on Equations (19), (20), (22), (25)-(28) and our obtained parameters, we can now compare our model with the multi-band afterglow LCs. As shown in Figure 1, the whole optical/UV and X-ray (except the last few data points) LCs can be well described with our model ${ }^{8}$. Especially in the optical/UV band, our model successfully explained the initial frequency-dependent decay feature and the corresponding spectral evolution. Besides the two achromatic breaks $t_{\mathrm{b}, 1}$ and $t_{\mathrm{b}, 2}$, there is an chromatic break $t_{\nu}$ in the optical/UV LCs. It denotes the time that $\nu_{\mathrm{b}}$ crosses an observational frequency $\nu$. For $\nu=R, V, B, U, U V W 1, U V M 2, U V W 2$, the corresponding breaks are $t_{\nu}=26.8,21.1,16.1,12.0,8.4,7.0,5.8 \mathrm{ks}$. The optical/UV LCs show a plateau between $t_{\nu}$ and $t_{\mathrm{b}, 1}$ with the same slope as the X-ray plateau; before $t_{\nu}$, the LCs rise with a slope of $(-0.32)$. It should be noted that

\footnotetext{
8 The initial steep decay of the X-ray LC before about $500 \mathrm{~s}$ is likely the prompt emission tail (M07) which is not a concern of our model.
} 


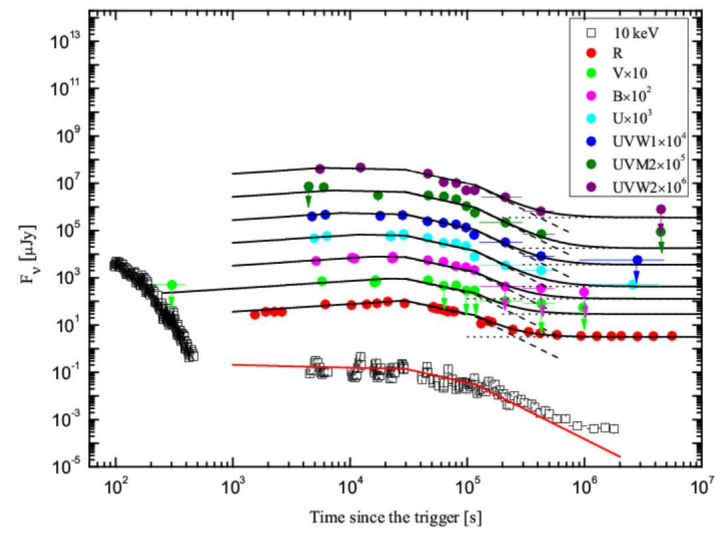

Figure 1. Theoretical LCs as compared with the multiband afterglow observations of GRB 060614. The $10 \mathrm{keV}$ unabsorbed X-ray data (empty squares) are downloaded from http://www.swift.ac.uk/burst_analyser/00214805/ (Evans et al. 2007, 2009). The $R$-band data (red filled circles) are taken from Della Valle et al. (2006) and Gal-Yam et al. (2006), while the optical/UV data in other bands are take from M07. The optical/UV data have been corrected for Galactic and host galaxy extinction with $A_{V, \mathrm{G}}=0.07$ and $A_{V, \mathrm{~h}}=0.05$, respectively. For clarity, the shown flux densities in the $V, B, U, U V W 1, U V M 2$ and $U V W 2$ bands have been rescaled by factors $10,10^{2}, 10^{3}$, $10^{4}, 10^{5}$ and $10^{6}$, respectively. The red solid line is our model predicted X-ray LC. The modeled optical/UV LCs are shown as the sum (black solid lines) of two components: the afterglow (black dashed lines) and the host (black dotted lines). The magnitude values of the host in each band are taken from M07. To produce the theoretical LCs, the parameters of $p_{1}=1.6, p_{2}=2.68, q=0.41, e=0.27$, $\epsilon_{\mathrm{e},-1}=1, \epsilon_{\mathrm{B},-2}=1.1 \times 10^{-2}, E_{\mathrm{f}, 52}=2.25, n_{0}=0.01$, $\xi_{4}=1.2, t_{\mathrm{f}}=29.7 \mathrm{ks}$ and $t_{\mathrm{j}}=117.2 \mathrm{ks}$ are used.

an exact calculation of afterglow radiation would give smooth spectral and temporal breaks (Granot \& Sari 2002), so such a chromatic break in the optical/UV LCs may not be clearly seen, especially when the data are sparsely sampled. Instead, the passage of $\nu_{\mathrm{b}}$ through the optical/UV band may show an average effect in the LCs: slowly rising at low frequencies and flattening at higher energies, just like the afterglow of GRB 060614 (M07). We emphasize, however, that our simple analytic model perfectly described this feature and no need to employ complicated numerical calculations. For the X-ray afterglow, we note that the data points after $\sim 10^{6} \mathrm{~s}$ obviously deviate from our modeling fit and suggest a late re-brightening or a flattening. M07 found that at the end the observations have small signal to noise ratios and approach the XRT sensitivity limit. We thus do not consider this inconsistency. There are also slight excesses between $2 \times 10^{5}$ and $10^{6} \mathrm{~s}$, this is because in our modeling we used the central value of 2.48 for the post-jet-break slope. When the uncertainty of this parameter is considered, this problem would be alleviated.

We conclude this subsection by comparing the $q$ value of GRB 060614 with that of GRB 091127. For GRB 091127, $q=0.64 \pm 0.08$ (Paper I), while GRB 060614 gives $q=0.41 \pm 0.20$. These results imply a similar evolution behavior of the injection break, with $q \sim 0.5$. However, at this stage it is premature to say that this represents a universal law of the injection break and more such events are needed to test this conjecture. We emphasize that the values of $q$ for both bursts are reliable, since the consistency checks have been performed with various afterglow observational constraints. Finally, we emphasize that this DPLH spectrum predicts an injection break frequency evolving as $\nu_{\mathrm{b}} \propto t^{-3(1+q) / 4}=t^{\sim(-1.1)}$ (for $\left.e=1\right)$, which is substantially faster than $\nu_{\mathrm{c}}$ in a single PL hard electron spectrum model. Therefore, when this kind of spectral break along with flat spectra in the optical band is observed in afterglows, it provides strong support to the above conjecture.

\section{2. $G R B 060908$}

The parameters to be constrained are $p_{1}, p_{2}, q, \epsilon_{\mathrm{e}}$, $\epsilon_{\mathrm{B}}, \xi, E$ and $A_{*}$. The observed spectral indices require $\nu_{\mathrm{m}}<\nu_{\mathrm{optNIR}}<\min \left(\nu_{\mathrm{b}}, \nu_{\mathrm{c}}\right)<\nu_{\mathrm{X}}$, then we have $p_{1}=$ $2 \beta_{\text {optNIR }}+1=1.66_{-0.58}^{+0.50}$ and $p_{2}=2 \beta_{\mathrm{X}}=2.34_{-0.44}^{+0.50}$. The value of $q$ can in principle be determined by the observed $\mathrm{X}$-ray decay index $\alpha_{\mathrm{X}}$. According to Equation (28), we get $q=4\left(\alpha_{\mathrm{X}}-p_{2} / 2\right) /\left(p_{2}-2\right)=-0.6_{-5.8}^{+9.0}$. It is not strange that $q$ is badly constrained, since $\alpha_{\mathrm{X}}$ has a very weak dependence on $q$ and it is mainly determined by $p_{2}$ which has large uncertainties. Based on the results of Subsection 4.1, we assume $q=0.5$ for GRB 060809 and test whether it is consistent with other observational properties. With this value of $q$ and $\alpha_{\mathrm{X}}=1.12$, we obtain $p_{2}=2.2$. Given that $p_{1}$ and $p_{2}$ obtained from the spectral indices have relatively large uncertainties, for simplicity we adopt $p_{1}=1.6$ and $p_{2}=2.2$ in the following calculations.

To calculate the flux density $F_{\nu}$, we should first determine the order between $\nu_{\mathrm{b}}$ and $\nu_{\mathrm{c}}$. Below we give some arguments: (i) the spectral analysis of Covino et al. (2010) requires $\nu_{\mathrm{b}}(8000 \mathrm{~s}) \gtrsim 5.5 \times 10^{14} \mathrm{~Hz}$, with $\nu_{\mathrm{b}} \propto$ $t^{-1.25}$ we have $\nu_{\mathrm{b}}(80 \mathrm{~s}) \gtrsim 0.7 \mathrm{keV}$; (ii) at the end of the $\mathrm{X}$-ray observations, $\nu_{\mathrm{c}}$ should not have crossed the Xray band. We simply require $\nu_{\mathrm{c}}\left(5 \times 10^{5} \mathrm{~s}\right) \lesssim 1 \mathrm{keV}$, with $\nu_{\mathrm{c}} \propto t^{1 / 2}$ we get $\nu_{\mathrm{c}}(80 \mathrm{~s}) \lesssim 3.0 \times 10^{15} \mathrm{~Hz}$. That is, at the beginning of the observations $(\sim 80 \mathrm{~s}), \nu_{\mathrm{b}}$ should be near the low-end of the XRT band, while $\nu_{\mathrm{c}}$ should be near the high-end of the ultraviolet band, i.e., $\nu_{\mathrm{m}}<\nu_{\mathrm{optNIR}}<$ $\nu_{\mathrm{c}}<\nu_{\mathrm{b}}<\nu_{\mathrm{X}}$. As $\nu_{\mathrm{b}}$ decreases and $\nu_{\mathrm{c}}$ increases, the 
spectrum transits to $\nu_{\mathrm{m}}<\nu_{\mathrm{optNIR}}<\nu_{\mathrm{b}}<\nu_{\mathrm{c}}<\nu_{\mathrm{X}}$ and eventually becomes $\nu_{\mathrm{m}}<\nu_{\mathrm{b}}<\nu_{\mathrm{optNIR}}<\nu_{\mathrm{c}}<\nu_{\mathrm{X}}$.

Since we have $\nu_{\mathrm{X}}>\nu_{\mathrm{c}}$ throughout the observations, the corresponding electrons may suffer from significant inverse Compton losses, especially when $\epsilon_{\mathrm{B}}$ has very small values. When the synchrotron self-Compton (SSC) effect is considered, the cooling frequency $\nu_{\mathrm{c}}$ would be reduced by a factor of $(1+Y)^{-2}$ and the Xray flux would be suppressed by $(1+Y)^{-1}$, here $Y$ is the Compton parameter (Sari \& Esin 2001). With the adopted parameters, we derive the break frequencies according to Equations (11)-(13) and replace $\nu_{\mathrm{c}}$ with $\nu_{\mathrm{c}}(1+Y)^{-2}$, i.e.

$$
\begin{aligned}
\nu_{\mathrm{m}} & \left.=3.4 \times 10^{7} \xi_{4}^{-1.33} \epsilon_{\mathrm{e},-1}^{3.33} \epsilon_{\mathrm{B},-4}^{1 / 2} E_{52}^{0.67} A_{*}^{-0.17} t_{\mathrm{d}}^{-1.67} \mathrm{H} \notin 33\right) \\
\nu_{\mathrm{b}} & =7.8 \times 10^{13} \xi_{4}^{2} \epsilon_{\mathrm{B},-4}^{1 / 2} E_{52}^{0.25} A_{*}^{0.25} t_{\mathrm{d}}^{-1.25} \mathrm{~Hz} \\
\nu_{\mathrm{c}} & =3.9 \times 10^{16} \epsilon_{\mathrm{B},-4}^{-3 / 2} E_{52}^{1 / 2} A_{*}^{-2} t_{\mathrm{d}}^{1 / 2}(1+Y)^{-2} \mathrm{~Hz} .
\end{aligned}
$$

The Compton parameter can be estimated as follows (RB08). For $\nu_{\mathrm{m}} \leqslant \nu_{\mathrm{c}} \leqslant \nu_{\mathrm{b}}$,

$$
\begin{aligned}
Y & \approx \frac{\nu_{\mathrm{b}}^{\mathrm{IC}} F_{\nu_{\mathrm{b}}}^{\mathrm{IC}}}{\nu_{\mathrm{b}} F_{\nu_{\mathrm{b}}}} \\
& =2 \gamma_{\mathrm{b}} \gamma_{\mathrm{c}} \zeta\left(\frac{\gamma_{\mathrm{m}}}{\gamma_{\mathrm{b}}}\right)^{p_{1}-1} \\
& =670 \epsilon_{\mathrm{e},-1} \epsilon_{\mathrm{B},-4}^{-1}(1+Y)^{-1},
\end{aligned}
$$

where $\zeta \equiv F_{\nu, \text { max }}^{\mathrm{IC}} / F_{\nu, \max }=n \sigma_{\mathrm{T}} r, F_{\nu_{\mathrm{b}}}$ and $F_{\nu_{\mathrm{b}}}^{\mathrm{IC}}$ are the synchrotron and SSC flux at $\nu_{\mathrm{b}}$, respectively, and $\nu_{\mathrm{b}}^{\mathrm{IC}} \simeq 2 \gamma_{\mathrm{b}}^{2} \nu_{\mathrm{b}}$. We note that $Y$ is only a simply function of $\epsilon_{\mathrm{e}}$ and $\epsilon_{\mathrm{B}}$. As long as $\epsilon_{\mathrm{B}} \lesssim 0.067 \epsilon_{\mathrm{e},-1}$, we have $Y \gtrsim 1$. Small values of $\epsilon_{\mathrm{B}} \lesssim 0.01$ are required for $\mathrm{GeV}$-detected bursts if the $\mathrm{GeV}$ emission arises from external shocks (e.g., Kumar \& Barniol Duran 2009, 2010; Beniamini et al. 2015), and are also supported by recent systematic studies using X-ray/optical (Santana et al. 2014) or radio (Barniol Duran 2014) afterglow observations. Such small values of $\epsilon_{\mathrm{B}}$ imply a large $Y$ and significant SSC losses, which have important effects on the derived blastwave energy and thus the prompt emission efficiency (Beniamini et al. 2015, 2016). With this consideration, Equation (36) can be written as

$$
Y \approx 25.9 \epsilon_{\mathrm{e},-1}^{1 / 2} \epsilon_{\mathrm{B},-4}^{-1 / 2} .
$$

For $\nu_{\mathrm{m}} \leqslant \nu_{\mathrm{b}} \leqslant \nu_{\mathrm{c}}$

$$
\begin{aligned}
Y & \approx \frac{\nu_{\mathrm{c}}^{\mathrm{IC}} F_{\nu_{\mathrm{c}}}^{\mathrm{IC}}}{\nu_{\mathrm{c}} F_{\nu_{\mathrm{c}}}} \\
& =2 \gamma_{\mathrm{c}}^{2} \zeta\left(\frac{\gamma_{\mathrm{m}}}{\gamma_{\mathrm{b}}}\right)^{p_{1}-1}\left(\frac{\gamma_{\mathrm{b}}}{\gamma_{\mathrm{c}}}\right)^{p_{2}-1} \\
& \propto \zeta \gamma_{\mathrm{m}}^{0.6} \gamma_{\mathrm{b}}^{0.6} \gamma_{\mathrm{c}}^{0.8}(1+Y)^{-0.8},
\end{aligned}
$$

where $\gamma_{\mathrm{c}}^{\mathrm{IC}} \simeq 2 \gamma_{\mathrm{c}}^{2} \nu_{\mathrm{c}}$, and $F_{\nu_{\mathrm{c}}}$ and $F_{\nu_{\mathrm{c}}}^{\mathrm{IC}}$ are the synchrotron and SSC flux at $\nu_{\mathrm{c}}$, respectively. After a simple derivation, Equation (38) gives $Y \propto t^{-0.1}$.

The transition occurs at $t=t_{\mathrm{bc}}$, which can be obtained by solving $\nu_{\mathrm{b}}\left(t_{\mathrm{bc}}\right)=\nu_{\mathrm{c}}\left(t_{\mathrm{bc}}\right)$. We note that the value of $Y$ is basically a constant throughout the observations; including the volution effect would only flatten the X-ray LC by $t^{0.1}$ after $t_{\mathrm{bc}}$. Given that our calculation of $Y$ is not sufficiently accurate, we do not consider its evolution and simply use Equation (37) in the following parameter estimations. This simplification is also consistent with the fitting results of a constant PL decay of the X-ray LC (Covino et al. 2010).

We define the time at which $\nu_{\mathrm{b}}$ crosses a specified optical/NIR frequency as $t_{\mathrm{b}, \nu}$. For $t<t_{\mathrm{b}, \nu}$, we have $\nu_{\mathrm{m}}<\nu<\min \left(\nu_{\mathrm{b}}, \nu_{\mathrm{c}}\right)<\nu_{\mathrm{X}}$, then the optical/NIR flux density can be obtained from Equation (15),

$$
F_{\nu}=1.75 \xi_{4}^{-0.4} \epsilon_{\mathrm{e},-1} \epsilon_{\mathrm{B},-4}^{0.65} E_{52}^{0.7} A_{*}^{0.95} t_{\mathrm{d}}^{-1.0}\left(\frac{\nu}{\nu_{\mathrm{R}}}\right)^{-0.3} \mu \mathrm{Jy},
$$

where $\nu_{\mathrm{R}}$ is $R$-band frequency. For $t \geqslant t_{\mathrm{b}, \nu}$, we have $\nu_{\mathrm{m}}<\nu_{\mathrm{b}}<\nu<\nu_{\mathrm{c}}<\nu_{\mathrm{X}}$ and $F_{\nu}=F_{\nu}\left(t_{\mathrm{b}, \nu}\right)\left(t / t_{\mathrm{b}, \nu}\right)^{-1.37}$ according to Equation (16).

The $10 \mathrm{keV}$ flux can be derived from Equation (17), (35) and (37),

$F_{10 \mathrm{keV}}=2.88 \times 10^{-5} \xi_{4}^{0.2} \epsilon_{\mathrm{e},-1}^{1 / 2} \epsilon_{\mathrm{B},-4}^{0.55} E_{52}^{1.02} A_{*}^{0.025} t_{\mathrm{d}}^{-1.12} \mu \mathrm{Jy}$.

To obtain the remaining parameters, we use the following observational constraints: (i) $F_{\nu_{\mathrm{R}}}(500 \mathrm{~s})=$ $622 \mu \mathrm{Jy}^{9} ;$ (ii) $F_{10 \mathrm{keV}}(15.7 \mathrm{ks})=0.016 \mu \mathrm{Jy} ;$ (iii) $\nu_{\mathrm{b}}(80 \mathrm{~s}) \lesssim 1 \mathrm{keV}$ and $\nu_{\mathrm{b}}(8000 \mathrm{~s}) \gtrsim 5.5 \times 10^{14} \mathrm{~Hz}$. Using Equations (39) and (40), conditions (i) and (ii) give

$$
\begin{aligned}
\epsilon_{\mathrm{B},-4}^{1.75} A_{*} & =13953.0 \epsilon_{\mathrm{e},-1}^{-2} E_{52}^{-2.74} \\
\xi_{4} & =3.78 \times 10^{9} \epsilon_{\mathrm{e},-1}^{-2.5} E_{52}^{-5.1} \epsilon_{\mathrm{B},-4}^{-2.75} A_{*}^{-0.125} .
\end{aligned}
$$

Using Equation (34), condition (iii) gives

$$
0.36 \lesssim \xi_{4}^{2} \epsilon_{\mathrm{B},-4}^{1 / 2} A_{*}^{0.25} E_{52}^{0.25} \lesssim 0.5 .
$$

Interestingly, Equation (43) leads to very strict limits and we simply take

$$
\xi_{4}^{2} \epsilon_{\mathrm{B},-4}^{1 / 2} A_{*}^{0.25} E_{52}^{0.25}=0.45 .
$$

Like the case of GRB 060614, here we also adopt $\epsilon_{\mathrm{e},-1}=1$. We adopt a typical prompt emission efficiency

\footnotetext{
9 This value has been corrected with the same Galactic and host galaxy extinction $E(B-V)=0.03$ (Covino et al. 2010).
} 
of $\eta_{\gamma}=E_{\gamma, \text { iso }} /\left(E_{\gamma, \text { iso }}+E\right)=15 \%$ (Beniamini et al. 2016) for GRB 060908, this corresponds to $E_{52}=$ 35. By substituting these values in Equations (41), (42) and (44), we get $\epsilon_{\mathrm{B},-4}=6.7, A_{*}=0.03$ and $\xi_{4}=0.42$. We note the value of $\epsilon_{\mathrm{B}}$ is well consistent with the statistical results of Santana et al. (2014) and Barniol Duran (2014). It is also consistent with the results of Beniamini et al. (2016), who re-analyzed the prompt emission efficiency using X-ray afterglows and taken into account the SSC effect.

It is important to perform consistency checks of our model with the above parameters. According to Equations (33) and (35), we have (i) $\nu_{\mathrm{c}}(80 \mathrm{~s})=4.5 \times 10^{15} \mathrm{~Hz}$ and $\nu_{\mathrm{c}}\left(5 \times 10^{5} \mathrm{~s}\right)=1.5 \mathrm{keV}$; (ii) $\nu_{\mathrm{m}}(100 \mathrm{~s})=4.4 \times$ $10^{14} \mathrm{~Hz}$. That is, at the very beginning $\nu_{\mathrm{m}} \lesssim \nu_{\mathrm{opt}}<\nu_{\mathrm{c}}$ is satisfied, and at the end $\nu_{\mathrm{c}}$ is at the low-end of the XRT band. We note that at $100 \mathrm{~s}, \nu_{\mathrm{m}}$ is the same as the $R$-band frequency and this time should correspond to the peak of the LC. This seems to be inconsistent with the observations. However, as discussed below, the data points before $\sim 100$ s may be dominated by emission from the reverse shock. Therefore, our obtained parameters are fully compatible with the observations and our afterglow modeling of GRB 060908 is self-consistent.

Based on Equations (16), (34), (39), (40) and the obtained parameters, we can now compare our model with the multi-band afterglow data. As shown in Figure 2, the DPLH model can describe the afterglow rather well. The X-ray excesses between about 300 and $1000 \mathrm{~s}$ are likely due to a complex flaring activity and can be modeled with two Gaussian functions (Covino et al. 2010). A more detailed analysis of flaring activity in this and other events was performed by Chincarini et al. (2010). The predicted optical/NIR LCs initially decay as $t^{-1}$, then steepen to $t^{-1.37}$ at the frequency-dependent break time $t_{\mathrm{b}, \nu}$, which ranges from 9.8 to $29.3 \mathrm{ks}$ for the observed bands. These values are basically consistent with the fitting results $\left(\sim 10^{3}-10^{4} \mathrm{~s}\right)$ of Covino et al. (2010). Unfortunately, observationally this break time cannot be well constrained by the data, let alone its chromaticity predicted by our model. The starting points of the black solid lines denote the times at which $\nu_{\mathrm{m}}$ crosses the corresponding bands, ranging from $88 \mathrm{~s}$ to $199 \mathrm{~s}$, which could slightly change for different parameters adopted in the afterglow modeling. The R-band data before $\sim 100 \mathrm{~s}$ exhibit an obvious excess component which decays as $t^{\sim(-1.4)}$. The origin of this component is not clear. One possibility is that the early decay is a superposition of the decay phase $\left(F_{\nu} \propto t^{-3}\right.$ Kobayash \& Zhang 2003) of the reverse shock emission and the smooth peak of the forward shock emission. This scenario is also compitable with our afterglow modeling which concerns only the for- ward shock emission. Since the reverse shock component is not distinctly identified in the LC, the relevant physical parameters cannot be constrained. We thus do not consider this physical process in our afterglow modeling.

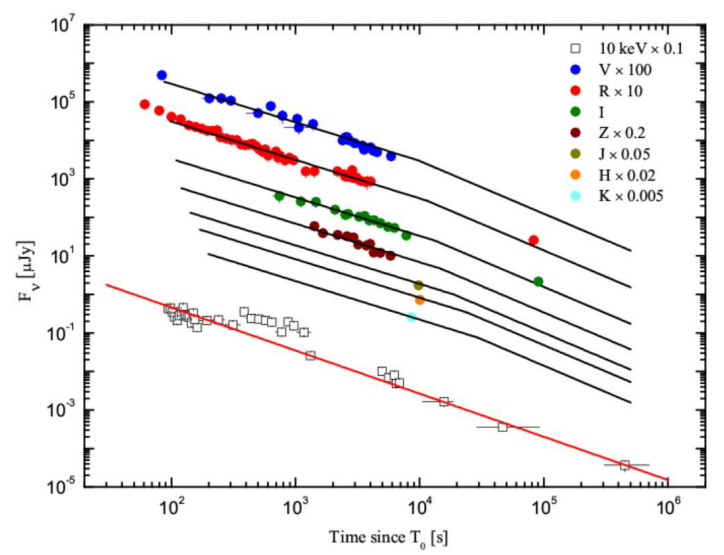

Figure 2. Theoretical LCs as compared with the multiband afterglow observations of GRB 060908. The $10 \mathrm{keV}$ unabsorbed X-ray data (empty squares) are downloaded from http://www.swift.ac.uk/burst_analyser/00228581/ (Evans et al. 2007, 2009). The optical/NIR data (filled circles) are taken from Covino et al. (2010). The optical/NIR data have been corrected with the same Galactic and host galaxy extinction $E(B-V)=0.03$. For clarity, the shown flux densities have been rescaled by factors ranging from 0.005 to 100 . The red and black solid lines are our afterglow modeling for the X-ray and the optical/NIR data, respectively. To produce the theoretical LCs, the parameters of $p_{1}=1.6, p_{2}=2.2, q=0.5, \epsilon_{\mathrm{e},-1}=1, \epsilon_{\mathrm{B},-4}=6.7, E_{52}=35$, $A_{*}=0.03$ and $\xi_{4}=0.42$ are used.

\section{CONCLUSION AND DISCUSSION}

The evidence for a DPLH spectrum in GRB afterglows have remained rare and somewhat ambiguous. Paper I showed that GRB 091127 gave strong evidence for the existence of a DPLH spectrum with an injection break assumed as $\gamma_{\mathrm{b}} \propto \gamma^{q}$ in the highly relativistic regime. In this work, we show that GRB 060614 and GRB 060908 provide further evidence for such a spectrum. We model the multi-band afterglow of GRB 060614 with the DPLH model in an ISM medium by taking into account a continuous energy injection process, while for GRB 060908, a wind-like circumburst density profile is employed. The evidence for a DPLH spectrum is strong in the case of GRB 060614 since we directly see a spectral break passing through the optical/UV band, while in the case of GRB060908 the evidence appears to be less strong. Perhaps most importantly, these bursts suggest a similar behavior in the evolution of the injection break, with $q \sim 0.5$. Whether this represents a universal law of the 
injection break remains uncertain and more such afterglow observations are needed to test this conjecture.

Below we give some discussions on the afterglow modeling of GRB 060614:

Firstly, we mention the work of Xu et al. (2009) who also modeled the multi-band afterglow of this burst. Different from our model, they used a standard $(p>2)$ electron energy spectrum and interpreted the observed spectral break as $\nu_{\mathrm{m}}$. This model was motivated by their SED analysis results at around $16 \mathrm{ks}$ : the SED from the optical to X-ray bands was fitted by a broken PL which gave $\beta_{\text {opt }}=-0.1 \pm 0.4$ (90\% CL)and $\beta_{\mathrm{X}}=0.9 \pm 0.1(90 \% \mathrm{CL})$. This spectrum is compatible with $\nu_{\mathrm{opt}}<\nu_{\mathrm{m}}<\nu_{\mathrm{X}}<\nu_{\mathrm{c}}$ in the standard afterglow model. When an energy injection is assumed, this model can describe the afterglow LCs rather well (Xu et al. 2009). However, we should note that the value of $\beta_{\text {opt }}$ cannot be well constrained in their fitting. Alternatively, the authors also fitted this SED by fixing $\beta_{\mathrm{X}}=\beta_{\text {opt }}+0.5$ and gave $\beta_{\text {opt }} \simeq 0.36$ and $\beta_{\mathrm{X}} \simeq 0.86$. These values are remarkably consistent with those of M07. Both works actually favor a positive $\beta_{\text {opt }}$ which is not compatible with $\nu_{\mathrm{opt}}<\nu_{\mathrm{m}}$. Therefore, their model has difficulties in explaining the early flat spectra in the optical/UV band.

Secondly, our model assumes an additional energy injection process, we now discuss its possible origins. The energy injection can be provided by the central engine, e.g., a rapidly spinning, strongly magnetized neutron star (the so-called "millisecond magnetar"; e.g., Usov 1992; Thompson 1994; Dai \& Lu 1998; Zhang \& Mészáros 2001; Zhang et al. 2006; Rowlinson et al. 2013; Gompertz et al. 2014). However, the simplest dipole spin-down model predicts $e=0$ that is not consistent with our obtained $e \sim 0.27$. Modifications to the simplest model are needed for this burst. Alternatively, such an energy injection can be provided by the soft tail of the outflow by considering that the extended emission is several times more energetic than the initial hard pulse. To check the consistency with our obtained parameters in Subsection 4.1, we assume the energy injection takes place at $t_{\mathrm{i}}=100 \mathrm{~s}$ and take $t_{\mathrm{f}}=29.7 \mathrm{ks}, E_{\mathrm{f}}=2.25 \times 10^{52} \mathrm{erg}$, then the initial kinetic energy of the outflow is $E_{\mathrm{i}}=E_{\mathrm{f}}\left(t_{\mathrm{i}} / t_{\mathrm{f}}\right)^{(1-e)} \sim$ $3.5 \times 10^{50} \mathrm{erg}$. Since the isotropic energy of the initial pulse is also $\sim 3.5 \times 10^{50}$ erg (M07), this corresponds a radiation efficiency of $\sim 50 \%$, which is consistent the median value of short GRBs (Fong et al. 2015).

Finally, the steep post-jet-break decay of GRB 060614 is due to significant sideways expansion of the jet based on the theory of Rhoads (1999). However, our derived bulk Lorentz factor at the jet-break time is $\sim 11$ that is highly relativistic. This is in conflict with the results given by numerical simulations and more sophisticated analytical treatments which suggest that the sideways expansion of a relativistic jet is not important until $\gamma$ drops below $\sim 2$ (Huang et al. 2000; Granot et al. 2001; Kumar \& Granot 2003; Cannizzo et al. 2004; Zhang \& MacFadyen 2009; de Colle et al. 2012; Granot \& Piran 2012; van Eerten \& MacFadyen 2012). There are also numerical works (e.g., Wygoda et al. 2011) supporting the simple analytic solutions of Rhoads (1999). Nevertheless, Granot \& Piran (2012) found that exponential sideways expansion can only occur for jets with extremely narrow initial half-opening angle $\left(\theta_{0} \ll 0.05\right)$ when $\gamma \lesssim 1 / \theta_{\mathrm{j}}$ is satisfied. Considering that the realistic GRB jets may have much more complicated hydrodynamical evolutions than employed in the above analytic and numerical models, whether an early exponential sideways expansion phase exists for typical jet opening angles still remains uncertain. Observationally, a fraction of X-ray afterglows show a jetbreak-like feature at around 1 day with post-break slope of $\sim p$ (e.g., Zhang et al. 2006; Willingale et al. 2007; Liang et al. 2008; Evans et al. 2009; Racusin et al. 2009; Panaitescu \& Vestrand 2012); some recent systematic studies of multi-band afterglows have also shown that a small fraction of GRBs have such jet-break features, simultaneously in X-rays and in the optical band (e.g., Fong et al. 2015; Li et al. 2015; Wang et al. 2015). Therefore, at least for some GRBs, sideways expansion should be significant when $\gamma \lesssim 1 / \theta_{\mathrm{j}}$ is satisfied, even though the jet is still in the highly relativistic regime.

Unlike GRB 060614 and GRB 091127 that clearly show both spectral breaks passing through the optical bands and chromatic evolutions in the multi-band LCs, the afterglow of GRB 060908 exhibits no such features. Such spectral breaks were interpreted as the injection break frequency $\left(\nu_{\mathrm{b}}\right)$ and the corresponding chromatic breaks were due to the passage of $\nu_{\mathrm{b}}$ through the optical bands. For GRB 060908, our model predicts that $\nu_{\mathrm{b}}$ crosses the observed optical bands between 9.8 and $29.3 \mathrm{ks}$, at which the chromatic breaks should be seen. Unfortunately, the data around this time are not sufficient to perform detailed spectral and temporal analysis. Nevertheless, the fact that the optical LCs show marginal evidence for a break at around $10^{3}-10^{4} \mathrm{~s}$ with a consistent post-break slope with our model prediction provides an additional support to the DPLH model.

Last but not least, we want to give a discussion on the value of $q$. In the original work of Bhattacharya \& Resmi (2004), the authors assumed the injection break to be the minimum electron Lorentz factor that can be accelerated by relativistic shocks, i.e., 
$\gamma_{\mathrm{b}} \equiv \gamma_{\mathrm{acc}}=\left(m_{\mathrm{p}} / m_{\mathrm{e}}\right) \gamma$; between $\gamma_{\mathrm{m}}$ and $\gamma_{\mathrm{b}}$, some other acceleration mechanisms take place and produce a hard electron spectrum. This means $q=1$ in their model. However, our work suggests $q \sim 0.5$ that disfavors this scenario. Moreover, the value of $\xi$ we derived is much larger than $m_{\mathrm{p}} / m_{\mathrm{e}}$. RB08 extended this function by assuming $\gamma_{\mathrm{b}} \propto(\beta \gamma)^{q}$ and attempted to find evidence by modeling the afterglows of three pre-Swift GRBs. Although their model can explain the afterglow LCs, the evidence for a DPLH spectrum is far from robust. First, no bursts in their sample show very flat spectra in the optical band. Their spectral indices are in the range of 0.6-0.9 that is typical for optical afterglows (e.g., Li ea al. 2012). Second, no spectral evolution was seen in their sample. That is, the injection break frequency $\nu_{\mathrm{b}}$ was actually not observed directly. Finally, for these bursts, the DPLH model is not the sole explanation. The LCs can also be reproduced by a model assuming continuous energy injection (e.g., Björnsson et al. 2002). Besides, their derived model parameters are much different from ours (see Paper I for a detailed discussion). Especially on the value of $q$, they gave $q \gtrsim 1$ for all bursts, while ours is substantially smaller. Since our works have provided the most robust evidence for a DPLH spectrum so far, $q \sim 0.5$ should be preferred.
The origin of the hard electron energy distribution is not clear. Our results may offer guidance in the right direction. Meanwhile, more observations of GRB afterglows with a hard electron spectrum and further developments in the area of simulations of the Fermi acceleration process in relativistic shocks will help us understand the origin of the observed spectra of GRBs and their afterglows.

We acknowledge the anonymous referee for helpful comments and suggestions. This work made use of data supplied by the UK Swift Science Data Centre at the University of Leicester. This study was supported by the Strategic Priority Research Program of the Chinese Academy of Sciences (Grant No. XDB23040400). SLX was also supported by the Hundred Talents Program of the Chinese Academy of Sciences (Grant No. Y629113). LMS acknowledges support from the National Program on Key Research and Development Project (Grant No. 2016YFA0400801) and the National Basic Research Program of China (Grant No. 2014CB845802).

\section{REFERENCES}

Achterberg, A., Gallant, Y. A., Kirk, J. G., \& Guthmann,

A. W. 2001, MNRAS, 328, 393

Andreev, M., Sergeev, A., Kurenya, A., et al. 2006, GCN, 5653,1

Antonelli, L. A., Covino, S., Testa, V., et al. 2006, GCN, 5546,1

Band, D., Matteson, J., Ford, L., et al. 1993, ApJ, 413, 281

Baring, M. G. 2004, NuPhS, 136, 198

Barniol Duran, R. 2014, MNRAS, 442, 3147

Barthelmy, S. D., Barbier, L. M., Cummings, J. R., et al. 2005, SSRv, 120, 143

Barthelmy, S., Barbier, L., Cummings, J., et al. 2006, GCN, 5256,1

Bednarz, J., \& Ostrowski, M. 1998, PhRvL, 80, 3911

Beniamini, P., Nava, L., Barniol Duran, R., \& Piran, T. 2015, MNRAS, 454, 1073

Beniamini, P., Nava, L., \& Piran, T. 2016, MNRAS, 461, 51

Beniamini, P., \& van der Horst, A. J. 2017, MNRAS, 472, 3161

Bhattacharya, D. 2001, BASI, 29, 107
Bhattacharya, D., Resmi, L. 2004, in ASP Conf. Ser. 312, Evolution of an Afterglow with a Hard Electron

Spectrum, ed. M. Feroci et al. (San Francisco, CA: ASP), 411

Björnsson G., Hjorth J., Pedersen K., \& Fynbo J. U. 2002, ApJL, 579, L59

Blandford, R. D., \& McKee, C. F. 1976, PhFl, 19, 1130

Burrows, D. N., Hill, J. E., Nousek, J. A., et al. 2005, SSRv, 120, 165

Cannizzo, J. K., Gehrel, N., \& Vishniac, E. T. 2004, ApJ, 601,380

Chevalier, R. A., \& Li, Z.-Y. 2000, ApJ, 536, 195

Chincarini, G., Mao, J., Margutti, R., et al. 2010, MNRAS, 406, 2113

Covino S., Campana S., Conciatore M. L., et al. 2010, A\&A, 521, A53

Curran, P. A., Evans, P. A., de Pasquale, M., Page, M. J., \& van der Horst, A. J. 2010, ApJL, 716, L135

Curran, P. A., Starling, R. L. C., van der Horst, A. J., \& Wijers, R. A. M. J. 2009, MNRAS, 395, 580

Dai, Z. G., \& Cheng, K. S. 2001, ApJL, 558, L109

Dai, Z. G., \& Lu, T. 1998, A\&A, 333, L87 
de Colle, F., Ramirez-Ruiz, E., Granot, J., \&

Lopez-Camara, D. 2012, ApJ, 751, 57

Della Valle, M., Chincarini, G., Panagia, N., et al. 2006, Natur, 444, 1050

Evans, P. A., Barthelmy, S. D., Beardmore, A. P., et al. 2006, GCN, 5544, 1

Evans, P. A., Beardmore, A. P., Page, K. L., et al. 2007, A\&A, 469, 379

Evans, P. A., Beardmore, A. P., Page, K. L., et al. 2009, MNRAS, 397, 1177

Fermi, E. 1954, ApJ, 119, 1

Filgas, R., Greiner, J., Schady, P., et al. 2011, A\&A, 537, A57

Fong, W., Berger, E., Margutti, R., \& Zauderer, B. A. 2015, ApJ, 815, 102

Frederiks, D. D., Hurley, K., Svinkin, D. S. 2013, ApJ, 779, 151

French, J., Melady, D., Hanlon, L., Jelínek, M., \& Kubánek, P. 2006, GCN, 5257, 1

Fugazza, D., Malesani, D., Romano, P., et al. 2006, GCN, 5276,1

Fynbo, J. P. U., Jakobsson, P., Prochaska, J. X., et al. 2009, ApJS, 185, 526

Fynbo, J. P. U., Watson, D., Thöne, C. C., et al. 2006, Natur, 444, 1047

Gal-Yam, A., Fox, D. B., Price, P. A., et al. 2006, Natur, 444, 1053

Gao, H., Lei, W. H., Zou, Y. C., Wu, X. F., \& Zhang, B. 2013, NewAR, 57, 141

Gehrels, N., Norris, J. P., Barthelmy, S. D., et al. 2006, Natur, 444, 1044

Golenetskii, S., Aptekar, R., Mazets, E. 2006, GCN, 5264, 1

Gompertz, B. P., O’Brien, P. T., \& Wynn, G. A. 2014, MNRAS, 438, 240

Granot, J., Miller, M., Piran, T., Suen, W. M., \& Hughes, P. A. 2001, in Gamma-ray Bursts in the Afterglow Era, Light Curves from an Expanding Relativistic Jet, ed. E. Costa, F. Frontera \& J. Hjorth, 312

Granot, J., \& Piran, T. 2012, MNRAS, 421, 570

Granot, J., \& Sari, R. 2002, ApJ, 568, 820

Holland, S. T. 2006, GCN, 5255, 1

Huang, Y. F., Gou, L. J., Dai, Z. G., \& Lu, T. 2000, ApJ, 543,90

Jarosik, N., Bennet, C. L., Dunkley, J., et al. 2011, ApJS, 192,14

Jin, Z. P., Li, X., Cano, Z., et al. 2015, ApJL, 811, L22

Kirk, J. G., Guthmann, A. W., Gallant, Y. A., \& Achterberg, A. 2000, ApJ, 542, 235

Kobayash, S., \& Zhang, B. 2003, ApJ, 497, 455

Kumar, P., Barniol Duran, R. 2009, MNRAS, 400, L75
Kumar, P., Barniol Duran, R. 2010, MNRAS, 409, 226

Kumar, P., \& Granot, J. 2003, ApJ, 591, 1075

Lemoine, M., \& Pelletier, G. 2003, ApJL, 589, L73

Li, L., Liang, E. W., Tang, Q. W., et al. 2012, ApJ, 758, 27

Li, L., Wu, X. F., Huang, Y. F. et al. 2015, ApJ, 805, 13

Liang, E. W., Racusin, J. L., Zhang, B., Zhang, B. B., \& Burrows, D. N. 2008, ApJ, 675, 528

Mangano, V., Holland, S. T., Malesani, D., et al. 2007, A\&A, 470, 105 (M07)

Melandri, A, Bernardini, M. G., D'Avanzo, P., et al. 2015, A\&A, 581, A86

Mészáros, P., \& Rees, M. J. 1993, ApJ, 405, 278

Mészáros, P., \& Rees, M. J. 1997, ApJ, 476, 232

Mészáros, P., \& Rees, M. J. 1999, MNRAS, 306, L39

Morgan, A. N., Vanden Berk, D. E., Brown, P., et al. 2006, GCN, 5553, 1

Nava, L., Vianello, G., Omodei, N., et al. 2014, MNRAS, 443,3578

Nousek, J. A., Kouveliotou, C., Grupe, D., et al. 2006, ApJ, 642,389

Nysewander, M., Reichart, D., Ivarsen, K., et al. 2006, GCN, 5545, 1

Palmer, D., Barbier, L., Barthelmy, S. D., et al. 2006, GCN, 5551, 1

Panaitescu, A., \& Kumar P. 2001, ApJ, 554, 667

Panaitescu, A., \& Kumar, P. 2002, ApJ, 571, 779

Panaitescu, A., \& Vestrand, W. T. 2012, MNRAS, 425, 1669

Parsons, A. M., Cummings, J. K., Gehrels, N. 2006, GCN, 5252,1

Price, P. A., Berger, E., \& Fox, D. B. 2006, GCN, 5275, 1

Racusin, J. L., Liang, E. W., Burrows, D. N., et al. 2009, ApJ, 698, 43

Rees, M. J., \& Mészáros, P. 1992, MNRAS, 258, 41

Rees, M. J., \& Mészáros, P. 1998, ApJL, 496, L1

Resmi, L., \& Bhattacharya, D. 2008, MNRAS, 388, 144 (RB08)

Rhoads, J. E. 1999, ApJ, 525, 737

Roming, P. W. A., Kennedy, T. E., Mason, K. O., et al. 2005, SSRv, 120, 95

Rowlinson, A., O'Brien, P. T., Metzger, B. D., Tanvir, N. R., \& Levan, A. J. 2013, MNRAS, 430, 1061

Santana, R., Barniol Duran, R., \& Kumar, P. 2014, ApJ, 785,29

Sari, R., \& Meśzaŕos, P. 2000, ApJL, 535, L33

Sari, R., \& Esin, A. A. 2001, ApJ, 548, 787

Sari, R., Piran, T., \& Halpern, J. P. 1999, ApJL, 519, L17

Sari, R., Piran, T., \& Narayan, R. 1998, ApJL, 497, L17

Shen, R., Kumar, P., \& Robinson, E. L. 2006, MNRAS, 371,1441 
Spitkovsky, A. 2008, ApJL, 682, L5

Starling, R. L. C., Van der Horst, A. J., Rol, E., et al. 2008, ApJ, 672, 433

Thompson, C. 1994, MNRAS, 270, 480

Troja, E., Sakamoto, T., Guidorzi, C., et al. 2012, ApJ, 761,50

Usov, V. V. 1992, Natur, 357, 472

van Eerten, H. J., \& MacFadyen, A. I. 2012, ApJ, 751, 155

Wang, X. G., Zhang, B., Liang, E. W., et al. 2015, ApJ, 219, 9

Wang, Y., Fan, Y. Z., Wei, D. M., \& Stefano, C. 2012, ChA\&A, 36, 148

Wiersema, K., Thöne, C. C., \& Rol, E. 2006, GCN, 5552, 1
Wijers R. A. M. J., \& Galama T. J. 1999, ApJ, 523, 177 Willingale, R., O'Brien, P. T., Osborne, J. P., et al. 2007, ApJ, 662, 1093

Wygoda, N., Waxman, E., \& Frail, D. 2011, ApJL, 738, L23

$\mathrm{Xu}$, D., Starling, R. L. C., Fynbo, J. P. U., et al. 2009, ApJ, 696, 971

Yang, B., Jin, Z. P., Li, X., et al. 2015, NatCo, 6, 7323

Zhang, B., Fan, Y. Z., Dyks, J., et al. 2006, ApJ, 642, 354

Zhang, B., \& Mészáros, P. 2001, ApJL, 552, L35

Zhang, B., Zhang, B. B., Liang, E. W., et al. 2007, ApJ, 655, L25

Zhang, Q., Huang, Y. F., \& Zong, H. S. 2015, ApJ, 811, 83 (Paper I)

Zhang, W., \& MacFadyen, A. 2009, ApJ, 698, 1261 